\title{
Gerência do Ciclo de Vida de VNFs e Implementação de Serviços Distribuídos na Rede
}

\author{
Giovanni Venâncio ${ }^{1}$ \\ Rogério Turchetti (Coorientador) ${ }^{2}$, Elias P. Duarte Jr. (Orientador) ${ }^{1}$ \\ ${ }^{1}$ Universidade Federal do Paraná (UFPR) - Curitiba - PR - Brasil \\ ${ }^{2}$ Universidade Federal de Santa Maria (UFSM) - Santa Maria - RS - Brasil \\ gvsouza@inf.ufpr.br, turchetti@redes.ufsm.br, elias@inf.ufpr.br
}

\begin{abstract}
Network Function Virtualization provides a flexible and cost-effective alternative to design and manage network services. In this work, a VNF Manager (VNFM) architecture was proposed to manage the VNF lifecycle. The proposed VNFM simplifies management operations and enables interoperability between platforms. The second contribution proposes VNF-Consensus, a VNF that consistently synchronizes the distributed control plane in an SDN network. The VNF decouples the synchronization mechanisms, avoiding the overhead on the controllers. Finally, we introduce AnyBone, a virtual backbone that provides broadcast services that are deployed on the network itself. AnyBone ensures the order of the messages by using a sequencer which was implemented as a VNF.
\end{abstract}

Resumo. A Virtualização de Funções de Rede oferece uma alternativa flexível para projetar e gerenciar serviços de rede. Neste trabalho foi proposta a arquitetura de um VNF Manager (VNFM) para o gerenciamento do ciclo de vida de VNFs. $O$ VNFM simplifica as operações de gerência e permite a interoperabilidade entre plataformas NFV. A segunda contribuição propõe a VNF-Consensus, uma VNF que sincroniza consistentemente o plano de controle distribuído em uma rede SDN. A VNF desacopla os mecanismos de sincronização, evitando a sobrecarga nos controladores. Por fim, a última contribuição é o AnyBone, um backbone virtual com serviços de difusão confiável e ordenada de mensagens implementados na própria rede. O AnyBone garante a ordem das mensagens através de um sequenciador, também implementado como uma VNF.

\section{Introdução}

A Virtualização de Funções de Rede (Network Function Virtualization - NFV) surge como uma alternativa para projetar e gerenciar serviços de rede, aplicando tecnologias de virtualização para implementar funções de rede em hardware de prateleira [Mijumbi et al. 2016]. Dessa forma, os serviços de rede são disponibilizados através de Funções Virtualizadas de Rede (Virtualized Network Functions - VNFs), simplificando a inclusão e alteração de novos serviços [Mijumbi et al. 2016].

Este trabalho apresenta um conjunto de contribuições que é classificado em duas partes: (i) gerenciamento de VNFs; (ii) implementação e execução de serviços distribuídos na rede. O VNF Manager (VNFM) é responsável principalmente pela gerência do ciclo de vida das VNFs [ETSI 2016]. As plataformas atuais que implementam o 
VNFM exigem uma grande quantidade de procedimentos, tornando-se complexas em sua utilização. Além disso, são pouco flexíveis, dificultando a integração entre diferentes tecnologias NFV. A primeira contribuição deste trabalho propõe a arquitetura de um VNFM para suprir estas deficiências, simplificando e flexibilizando as operações de gerência através de APIs que permitem gerenciar de maneira completa o ciclo de vida das VNFs. O VNFM proposto é utilizado na plataforma FENDE (http://coral.ufsm.br/gt-fende), uma Marketplace para a distribuição e execução de VNFs e SFCs (Service Function Chains).

As redes SDN (Software-Defined Network) separam o plano de controle do plano de dados [Kreutz et al. 2015]. Em geral, o plano de controle é centralizado, o que traz desafios em termos de disponibilidade, escalabilidade e desempenho [Canini et al. 2015]. Um dos maiores desafios é garantir a consistência das operações realizadas na rede que precisam ser consistentemente sincronizadas entre múltiplos controladores. A segunda contribuição deste trabalho é a VNF-Consensus, uma VNF responsável por garantir a consistência de operações em um plano de controle distribuído. Esta abordagem desacopla os controladores das tarefas de sincronização, diminuindo a sua carga de trabalho.

A difusão confiável é uma abstração importante para o desenvolvimento de aplicações distribuídas [Défago et al. 2004]. A difusão confiável não garante a ordem em que os processos entregam as mensagens, tornando-se necessária a difusão confiável e ordenada. Em geral, são implementadas na própria aplicação, aumentando a complexidade para o seu desenvolvimento [Li et al. 2016]. Assim, a última contribuição deste trabalho propõe o AnyBone: um backbone virtual baseado em NFV que oferece primitivas de difusão para garantir a entrega confiável e ordenada das mensagens. O AnyBone define a ordem das mensagens através de um sequenciador, disponibilizado através de uma VNF.

O restante deste trabalho está organizado da seguinte forma. A Seção 2 apresenta a especificação de um VNFM para o gerenciamento de VNFs. A Seção 3 descreve a VNFConsensus e a Seção 4 detalha o AnyBone. As conclusões são apresentadas na Seção 5.

\section{Simplificando o Gerenciamento do Ciclo de Vida de VNFs}

O padrão NFV do ETSI (European Telecommunications Standards Institute) [ETSI 2016] define o NFV-MANO (NFV MANagement and Orchestration), responsável por realizar a gerência das VNFs. Um dos principais módulos do bloco NFV-MANO é o VNF Manager (VNFM), responsável principalmente pela gerência do ciclo de vida das VNFs, que consiste primariamente da instanciação, remoção e atualização.

As plataformas que implementam o VNFM proposto pela ETSI [Tacker 2017, OpenBaton 2017, ETSI 2017] são complexas, uma vez que demandam múltiplos procedimentos para operar o ciclo de vida das VNFs. Além disso, tais soluções utilizam requisições REST, diminuindo a flexibilidade e dificultando a integração com outras tecnologias NFV, visto que cada solução implementa uma API diferente. Por fim, estas soluções não gerenciam as VNFs de maneira completa, demandando o gerenciamento manual das funções ou a utilização de ferramentas adicionais. Apesar da diversidade de plataformas que implementam as responsabilidades do NFV-MANO, nenhuma é totalmente adequada ao conjunto de operações previstas para este bloco.

Este trabalho propõe uma especificação para o VNFM com o objetivo de simplificar o gerenciamento do ciclo de vida das VNFs, abstraindo diversas operações e 
permitindo a interoperabilidade entre diferentes plataformas NFV. A Figura 1 mostra os principais componentes da arquitetura do VNFM proposto.

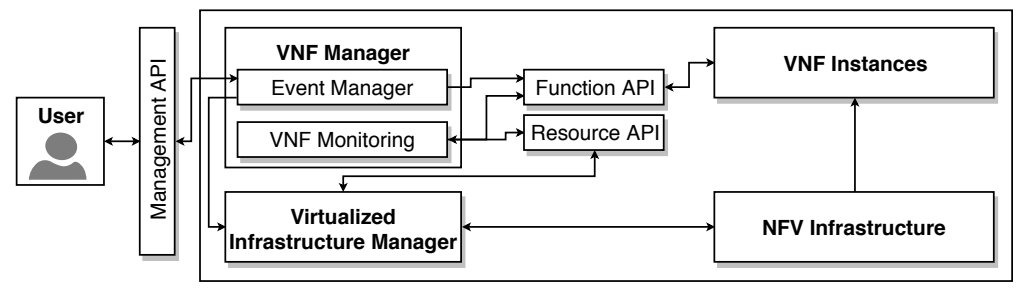

Figura 1. Arquitetura onde o VNFM está inserido.

Para prover a automação necessária foi proposto um módulo denominado Event Manager (EM), responsável por receber as requisições da camada de usuário através da Management API. As funcionalidades principais disponibilizadas pela Management API são: criação, remoção e atualização de VNFs. A partir destas requisições, o VNFM realiza, de maneira automática, todos os procedimentos para as operações de gerência. Para tanto, o EM comunica-se com duas outras APIs para simplificar a compatibilidade do VNFM. A Resource API define as funcionalidades para que o VNFM possa gerenciar os recursos das máquinas virtuais que irão hospedar as VNFs, enquanto que a Function API define as funcionalidades que o VNFM deve executar em nível de software.

Um protótipo da arquitetura foi implementado em Python e utiliza requisições do tipo REST para comunicação entre seus módulos. Neste trabalho, a Resource API foi implementada utilizando como base o Tacker [Tacker 2017], enquanto que para a Function API cada VNF disponibiliza uma interface REST.

\section{Avaliação Experimental}

Esta seção avalia as principais operações do protótipo executado em um servidor Dell PowerEdge com processador Intel Xeon(R) E3-1220v6 3.00GHz, com 4 núcleos, memória de 8GB e sistema operacional Ubuntu 16.04. Todos os testes foram realizados 30 vezes e os resultados são apresentados com um intervalo de confiança de $95 \%$.

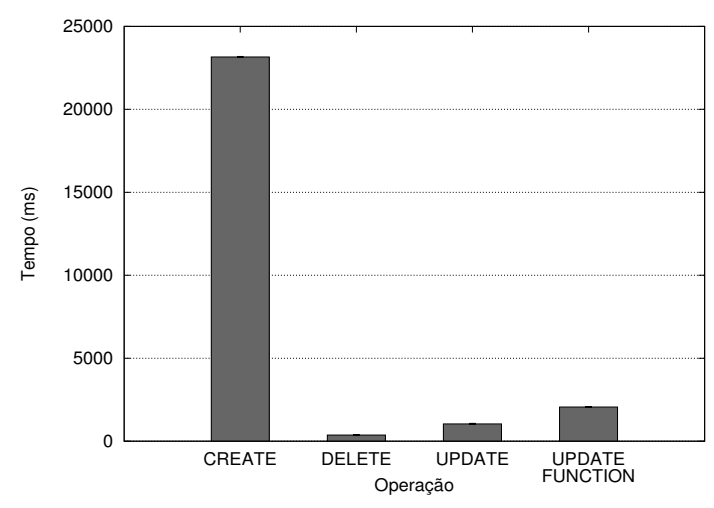

(a) Tempo total de execução das operações.

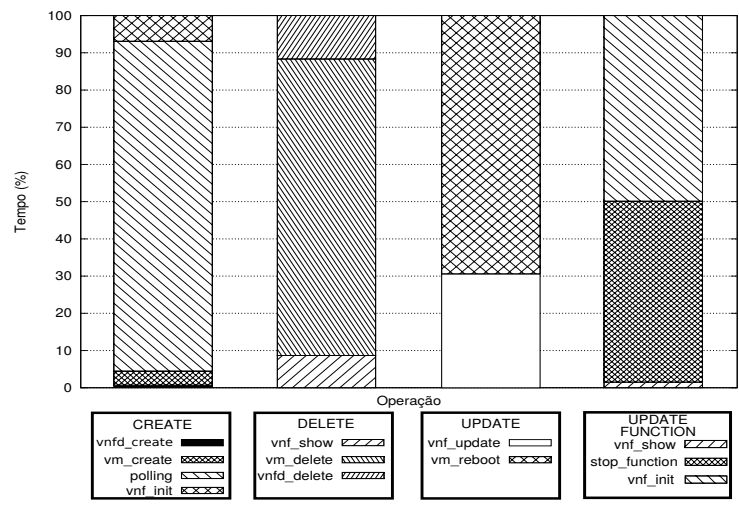

(b) Tempo de execução das suboperações.

Figura 2. Avaliação das operações de gerência.

A Figura 2(a) exibe o tempo das operações de gerência. A operação Create teve a maior duração devido ao tempo em que o sistema aguarda a criação da máquina virtual 
(polling) para então configurar a função de rede (vnf_init), conforme mostra a Figura 2(b). Além disso, o protótipo utiliza um máximo de 1,5\% de CPU e 0,25\% de memória, demonstrando ser uma solução eficaz para o gerenciamento do ciclo de vida das VNFs.

\section{Sincronização Consistente de um Plano de Controle SDN Distribuído}

O objetivo das redes SDN é separar o plano de controle do plano de dados [Kreutz et al. 2015]. Em geral, o plano de controle é centralizado [Ho et al. 2016], afetando a disponibilidade, escalabilidade e desempenho das redes SDN [Canini et al. 2015].

Em geral, o plano de controle é distribuído aplicando redundância [Schiff et al. 2016]. Entretanto, é necessário garantir a consistência de operações na rede, já que ações do plano de controle entre múltiplos controladores precisam ser sincronizadas [Schiff et al. 2016]. As soluções que desenvolvem um plano de controle SDN distribuído empregam os próprios controladores para realizar as tarefas de sincronização [Canini et al. 2015, Ho et al. 2016] ou sincronizam as ações da rede no plano de dados [Schiff et al. 2016, Dang et al. 2015]. No entanto, tais soluções oferecem alto custo computacional ou exigem mudanças no protocolo SDN ou nos próprios switches.

A segunda contribuição deste trabalho propõe uma VNF para manter a consistência de um plano de controle distribuído, sem aumentar o custo computacional dos controladores e sem modificar o plano de dados ou o protocolo SDN. A VNF, denominada de VNF-Consensus, implementa um algoritmo de consenso para manter a consistência no plano de controle SDN. Informalmente, o consenso permite que um conjunto de processos proponham valores iniciais diferentes e entrem em acordo sobre um valor final. Em particular, a VNF proposta é baseada no algoritmo de consenso Paxos [Lamport 1998].

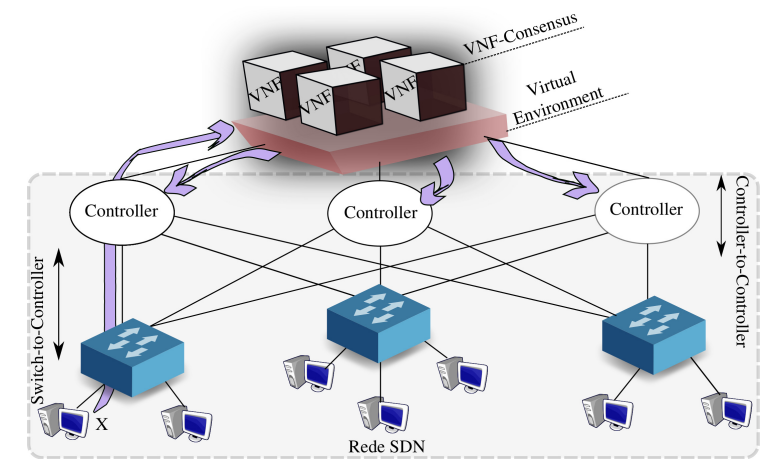

Figura 3. Uma rede SDN executando a VNF-Consensus.

A Figura 3 mostra a interação entre os controladores com a VNF-Consensus. Quando uma nova regra na rede precisa ser sincronizada, antes de ser instalada o respectivo controlador encaminha esta regra para a $V N F$-Consensus. A instância da $V N F$ Consensus recebe a regra, executa o algoritmo de consenso e retorna a decisão aos controladores, que por sua vez instalam a regra decidida. Note que este mecanismo não é bloqueante e o controlador continua atendendo novas requisições antes da decisão do consenso. Depois que uma decisão é tomada, todos os controladores recebem o resultado final e atualizam seu estado local, mantendo o plano de controle consistente. 


\section{Avaliação Experimental}

Nesta seção são apresentados os resultados que avaliam a VNF-Consensus em um servidor com processador AMD FX-4300 3.8 GHz de 4 núcleos e sistema operacional Ubuntu 16.04. Para criar a rede SDN foram utilizados três controladores Ryu ${ }^{1}$, três switches e o protocolo OpenFlow [McKeown et al. 2008]. A implementação do Paxos é baseada na libPaxos $^{2}$. A VNF-Consensus possui três instâncias, de forma a tolerar uma falha. Para cada experimento três amostras foram coletadas.

A Figura 4(a) mostra a utilização de CPU para diferentes cenários. Quando o controlador executa a sincronização o uso de CPU é em média 27\%. Ao utilizar a VNFConsensus a média do uso de CPU do controlador diminui para $14 \%$. Este experimento mostra claramente a vantagem de desacoplar a execução do consenso do controlador. Como consequência, a disponibilidade do controlador aumenta, uma vez que o custo de sincronização é realocado para uma entidade externa.

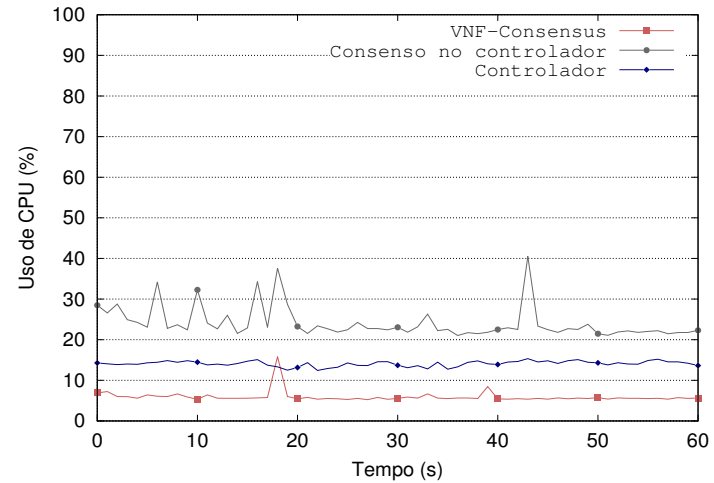

(a) Utilização de CPU.

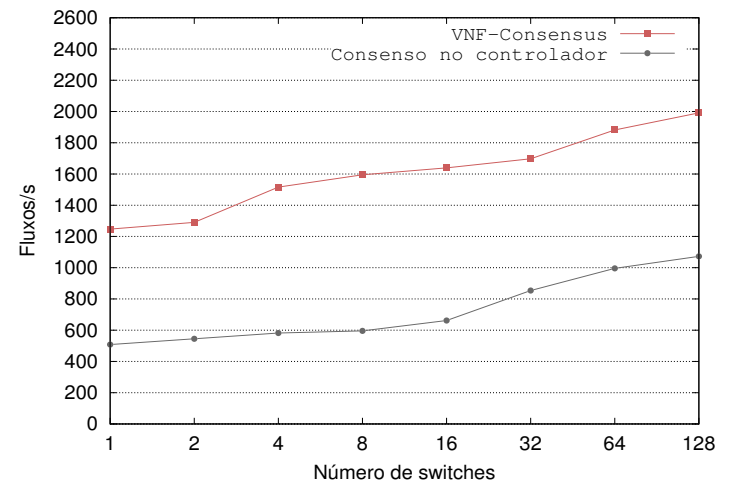

(b) Fluxos por segundo suportado pelo controlador.

Figura 4. Sincronização do plano de controle: avaliação do desempenho.

Devido a menor utilização de CPU, o experimento exibido na Figura 4(b) mostra que a VNF-Consensus aumenta o número de requisições no controlador em até $53 \%$. Além disso, quando os controladores estão sobrecarregados com outras tarefas, a $V N F$ Consensus aumenta a vazão do fluxo de regras instalado em 3,6 vezes. O mesmo ocorre quando o tamanho da rede aumenta: para até 10 controladores, verificou-se que a latência do consenso diminui em média $54 \%$ e obtém vazão 2,8 vezes maior.

\section{AnyBone}

Uma abstração importante para o desenvolvimento de aplicações distribuídas é a difusão confiável, que garante a entrega das mensagens por todos os processos corretos [Défago et al. 2004]. Além disso, um processo pode exigir que todas as mensagens sejam entregues em uma determinada ordem por todos os demais processos. Na prática, a implementação da difusão confiável e ordenada é feita na própria aplicação, consequentemente aumentando a complexidade para o seu desenvolvimento [Li et al. 2016].

Em geral, as plataformas e protocolos existentes que implementam a difusão confiável e ordenada executam nas máquinas dos usuários ([Hunt et al. 2010,

\footnotetext{
${ }^{1}$ https://osrg.github.io/ryu/

${ }^{2}$ https://bitbucket.org/sciascid/libpaxos
} 
Li et al. 2016]). Neste contexto é proposto o AnyBone, um backbone virtual que oferece difusão confiável e ordenada utilizando NFV em uma rede SDN. Em especial, é oferecida a difusão confiável, atômica, atômica FIFO e atômica causal.

\section{Difusão Confiável de Mensagens pelo AnyBone}

O algoritmo de difusão confiável implementado pelo AnyBone é descrito em [Chandra and Toueg 1996]. Para as difusões ordenadas é utilizado um sequenciador que considera duas sequências de mensagens. A primeira respeita a ordem local de transmissão de cada processo, possibilitando por exemplo, que a ordem FIFO seja implementada. A outra é a ordem global que garante que a ordem de entrega será atômica.

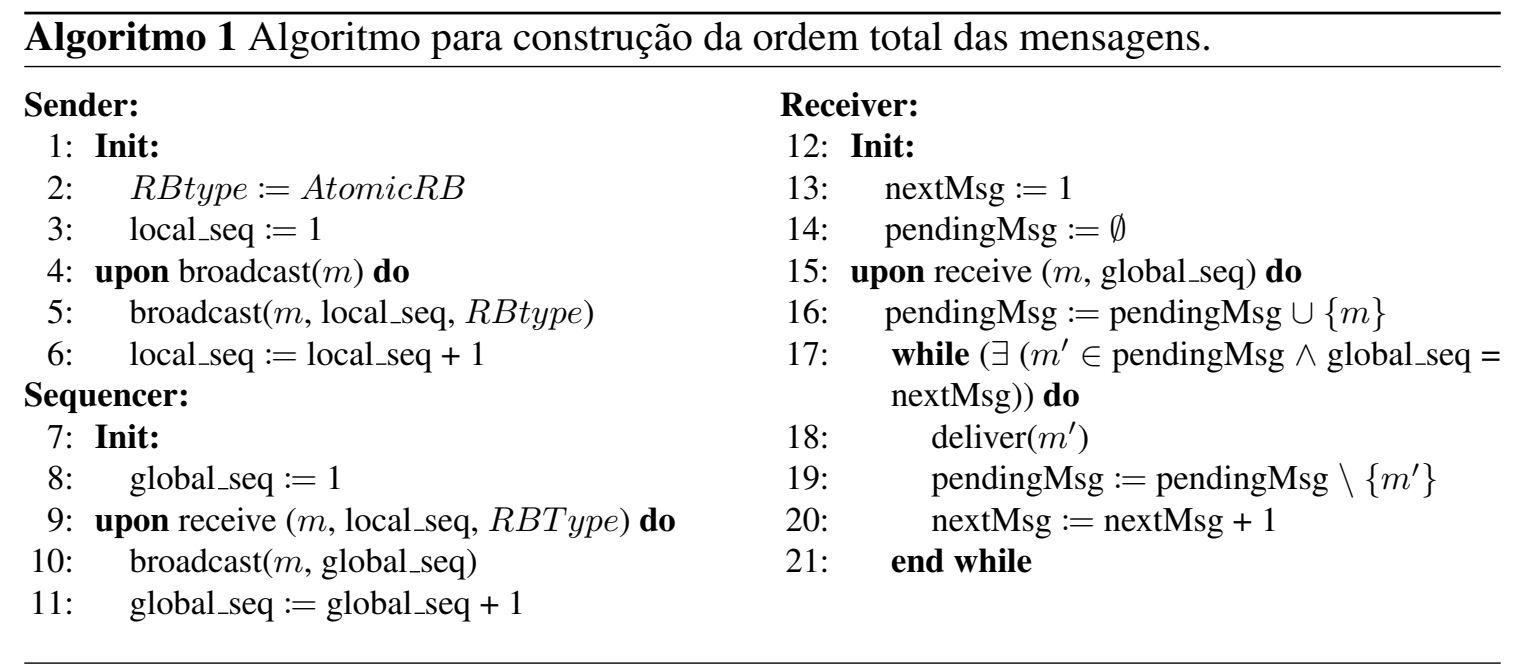

No Algoritmo 1 é apresentado o pseudo-código para a construção da ordem atômica entre os processos. Uma mensagem $m$ é transmitida por difusão carregando o contador local de mensagens e o tipo da difusão. O contador global é inserido pelo sequenciador na mensagem e incrementado sempre após a retransmissão de uma mensagem aos processos finais. Cada processo que recebe uma mensagem $m$, adiciona esta mensagem ao conjunto de mensagens pendentes (pending $M s g$ ). Logo após é feita a verificação se existe alguma mensagem $m^{\prime}$ do processo emissor que possui o contador igual ao identificador da próxima mensagem (nextMsg) e também se $m^{\prime}$ ainda está em pending $M s g$.

\section{Arquitetura e Implementação do AnyBone}

No AnyBone a ordem total das mensagens é garantida através de um sequenciador implementado como uma VNF, denominada de VNF-Sequencer, responsável por gerenciar toda a comunicação de forma a garantir as propriedades de comunicação definidas na aplicação distribuída. O AnyBone assume que o sequenciador nunca falha e considera-se um canal confiável. Para disponibilizar as primitivas de comunicação às aplicações, é oferecida uma API na biblioteca denominada de RBCast.

A arquitetura do AnyBone pode ser visualizada na Figura 5. Do funcionamento padrão do protocolo OpenFlow, toda mensagem que não possui entrada na tabela de fluxos é encaminhada ao controlador. Se o algoritmo escolhido for a difusão confiável, as mensagens não precisam ser enviadas ao sequenciador e são entregues de acordo com o algoritmo descrito em [Chandra and Toueg 1996]. Caso contrário, o controlador SDN é 


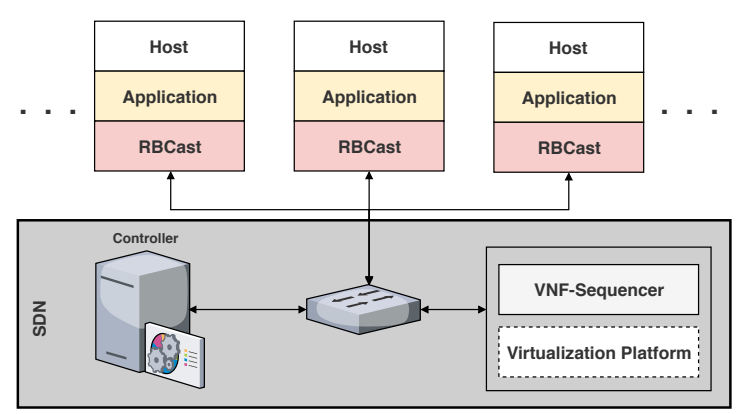

Figura 5. Arquitetura do AnyBone.

configurado para que o fluxo de pacotes seja encaminhado para a própria $V N F$-Sequencer. Ao receber o fluxo de pacotes, a VNF-Sequencer executa o algoritmo especificado pelo processo emissor e retransmite as mensagens aos processos receptores.

\section{Avaliação Experimental}

Esta seção avalia o desempenho da $V N F$-Sequencer em uma rede SDN. O sistema possui processador Intel Core i5-7200U@2.50GHz com 4 núcleos e sistema operacional Ubuntu 16.04. Para cada experimento são executadas 1000 difusões e os dados apresentados são valores médios de 10 amostras, utilizando um intervalo de confiança de $95 \%$.

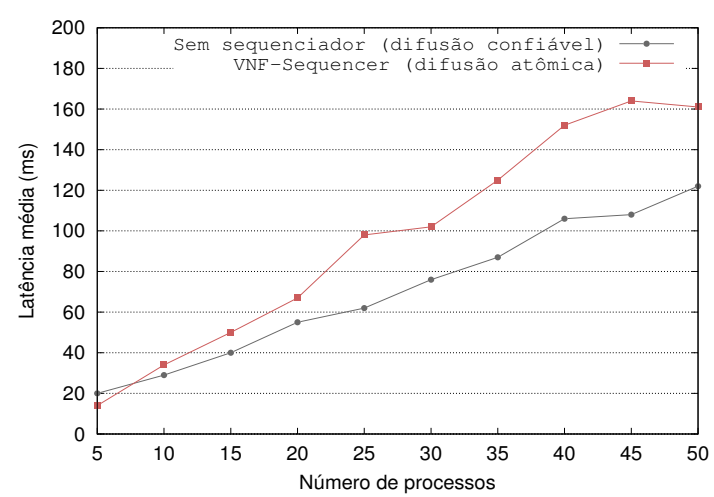

(a) Latência para a entrega das mensagens.

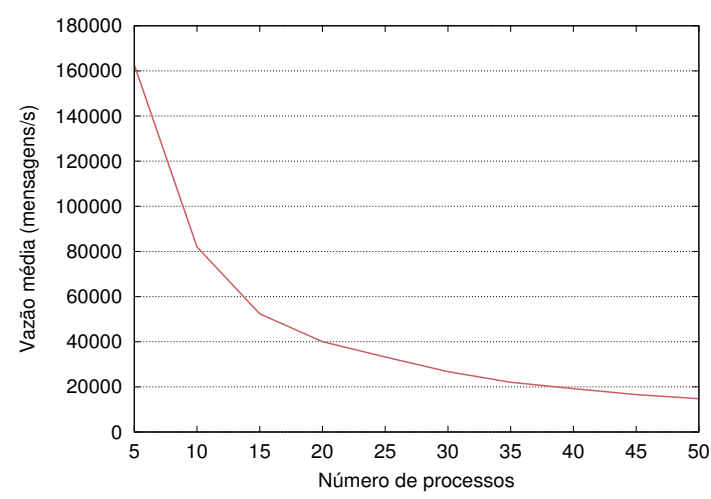

(b) Vazão para a difusão atômica.

Figura 6. VNF-Sequencer: Latência e Vazão.

A Figura 6(a) mostra que conforme o número de processos aumenta, a latência aumenta em média $32 \%$ com a VNF-Sequencer. Essa sobrecarga ocorre devido a VNFSequencer garantir a ordem total para todos os processos. Já o experimento da Figura 6(b) mostra que o AnyBone atinge uma vazão de 15000 mensagens/s na difusão atômica.

\section{Conclusão}

Este trabalho propôs um conjunto de contribuições no contexto de NFV, abordando a gerência do ciclo de vida das VNFs e a implementação de serviços distribuídos na própria rede. A primeira contribuição propõe a arquitetura de um VNFM que simplifica o gerenciamento do ciclo de vida das VNFs, de forma completa e a permitir o uso de diferentes plataformas. O VNFM proposto está sendo utilizado em um ambiente de produção na plataforma FENDE, uma Marketplace para a distribuição e execução de VNFs. 
A segunda contribuição descreve a VNF-Consensus, uma VNF que sincroniza um plano de controle distribuído em uma rede SDN. Essa VNF implementa o Paxos para evitar que os próprios controladores executem a sincronização. Por fim, é descrito o AnyBone, um backbone virtual que implementa uma VNF para oferecer múltiplos serviços de difusão. Destaca-se que este trabalho tem como contribuição original a implementação dentro da rede de serviços normalmente executados na camada de aplicação.

\section{Referências}

Canini, M., Kuznetsov, P., Levin, D., and Schmid, S. (2015). A distributed and robust SDN control plane for transactional network updates. In IEEE Conference on Computer Communications (INFOCOM).

Chandra, T. D. and Toueg, S. (1996). Unreliable failure detectors for reliable distributed systems. Journal of $A C M, 43(2)$.

Dang, H. T., Sciascia, D., Canini, M., Pedone, F., and Soulé, R. (2015). Netpaxos: Consensus at network speed. In Symposium on Software Defined Networking Research, (SOSR'15/SIGCOMM).

Défago, X., Schiper, A., and Urbán, P. (2004). Total order broadcast and multicast algorithms: Taxonomy and survey. ACM Comput. Surv., 36(4):372-421.

ETSI (2017). Open source mano. https: / / osm.etsi.org/. Accessed: 2017-11-21.

ETSI (Available at http://www.etsi.org/technologies-clusters/technologies/nfv, Accessed on October 02, 2016). Etsi gs nfv 002: Architectural framework.

Ho, C. C., Wang, K., and Hsu, Y. H. (2016). A fast consensus algorithm for multiple controllers in softwaredefined networks. In 18th International Conference on Advanced Communication Technology (ICACT).

Hunt, P., Konar, M., Junqueira, F. P., and Reed, B. (2010). Zookeeper: Wait-free coordination for internetscale systems. In USENIX annual technical conference, volume 8, page 9. Boston, MA, USA.

Kreutz, D., Ramos, F. M., Verissimo, P. E., Rothenberg, C. E., Azodolmolky, S., and Uhlig, S. (2015). Software-defined networking: A comprehensive survey. Proceedings of the IEEE, 103(1):14-76.

Lamport, L. (1998). The part-time parliament. ACM Transactions on Computer Systems (TOCS), 16(2).

Li, J., Michael, E., Sharma, N. K., Szekeres, A., and Ports, D. R. K. (2016). Just say no to paxos overhead: Replacing consensus with network ordering. In 12th USENIX Symposium on Operating Systems Design and Implementation (OSDI 16).

McKeown, N., Anderson, T., Balakrishnan, H., Parulkar, G., Peterson, L., Rexford, J., Shenker, S., and Turner, J. (2008). Openflow: enabling innovation in campus networks. ACM SIGCOMM Computer Communication Review, 38(2):69-74.

Mijumbi, R., Serrat, J., Gorricho, J.-L., Bouten, N., De Turck, F., and Boutaba, R. (2016). Network function virtualization: State-of-the-art and research challenges. IEEE Communications Surveys \& Tutorials, 18(1):236-262.

OpenBaton (2017). Openbaton. https: / / openbaton.github. io/. Accessed: 2017-11-21.

Schiff, L., Schmid, S., and Kuznetsov, P. (2016). In-Band Synchronization for Distributed SDN Control Planes. SIGCOMM Comput. Commun. Rev., 46(1).

Tacker (2017). Tacker. https: / /wiki. openstack.org/wiki/Tacker. Accessed: 2017-11-21. 